\title{
Improving FEM computations for the simulation of thermograms at the urban scale
}

\author{
José Pedro Aguerre ${ }^{1}$, Raphaël Nahon ${ }^{2}$, Eduardo Fernández ${ }^{1}$, Benoit Beckers ${ }^{2}$ \\ ${ }^{1}$ Centro de Cálculo, Universidad de la República. Montevideo, Uruguay \\ ${ }^{2}$ Urban Physics Joint Laboratory, I2S UPPA. Anglet, France
}

\begin{abstract}
Infrared cameras allow for the estimation of the temperature of urban surfaces. A time sequence of thermograms can be accurately simulated by computational techniques such as the finite element method (FEM) for transient heat transfer. The main objective of this work was to improve FEM convective and radiative boundary conditions, enabling a more precise analysis of an urban scene. The software Cast3m was used for performing the simulations, and the results were compared to a set of thermograms taken in the city of Bayonne through a clear-sky day. A street composed of two blocks was selected for the study, where the geometrical properties and boundary conditions were carefully established. The conducted experiments show that free internal air temperatures, rather than fixed, increase the realism of the simulation. Furthermore, the computational performance of the model was improved by exploiting specific properties of urban geometries.
\end{abstract}

\section{Introduction}

The analysis of thermograms of urban perspective views allows to obtain relevant information about the thermal behavior of buildings (Beckers and GarciaNevado (2018)). Heat transfer simulation engines bring more information into the studies, because a clean comparison between accurate simulations and experimental results can reveal details about the occupants behavior and the thermal comfort of the urban scenario. The analysis of this spatialized information can help in the redesign of city elements, and even on the projection of future buildings. Nevertheless, the simulations need to be as accurate as possible to perform a useful analysis.

The computational simulation of transient heat transfer involves dealing with several boundary conditions such as convection and radiation. Convection happens at both the exterior environment and interior spaces, having a significant effect on internal air masses and urban surfaces temperatures. On the other hand, radiation is emitted at different wavelengths by every surface with a temperature above the absolute zero. It can be studied at two levels: short wave radiation is emitted by the sun and refracted by the sky before reaching the urban surface, and long wave radiation is emitted and exchanged by the urban materials and the sky.

Thermal studies of urban elements are usually addressed using simplified models like electric circuit analogy methods, providing a balance between accuracy and algorithmic performance (Asawa et al. (2008); Kramer et al. (2012); Reinhart and Davila (2016)). Nevertheless, the spatialized information shown in thermograms is difficult to simulate with these kind of methods due to the high level of detail and granularity needed. Other computational techniques for dealing with finer meshes are available, such as finite difference methods (FDM) or FEM (Lewis et al. (2004)). These kind of methods are not specifically designed for cities, thus adaptations must be done (Lü (2002); Huttner and Bruse (2009); Idczak et al. (2010)). A careful mix between these highly accurate techniques and certain modeling simplifications can enable reliable studies to be tackled using regular PCs in reasonable execution times.

In this work, a study was conducted for improving FEM boundary conditions at the urban scale using the software Cast3m (Charras and Di Paola (2011)). Two problems were tackled. In the first place, a method inherited from previous electric analogy works (Fraisse et al. (2002); Nielsen (2005)) was adapted to FEM to address the interaction between the interior surfaces and internal air. Secondly, radiation computations, which are the most expensive part of the computations, were accelerated within Cast $3 \mathrm{~m}$, taking advantage of the low density factor (which is the rate between non-zero elements and the total number of elements) of the involved sparse form factors matrices (Aguerre et al. (2017)). These modifications were applied to simulate the test case presented in Aguerre et al. (2018), where a real thermography campaign was compared to FEM results. Higher accuracy is observed with the proposed modifications. 


\section{Methods}

In this section, FEM for transient heat transfer is described, and the two proposed modifications for improving the computation of boundary conditions are presented.

\section{Transient heat transfer}

The behavior of heat conduction can be expressed by a differential equation that relies on the heat conservation law to describe the transient heat flow:

$$
k \Delta T=\rho c_{p} \frac{\partial T}{\partial t}
$$

In this equation, $k\left(W m^{-1} K^{-1}\right)$ is the thermal conductivity of the material, considered constant along the solid and isotropic (equal in all directions). $\Delta$ is the spatial temperature laplacian. $\rho\left(\mathrm{kgm}^{-3}\right)$ and $c_{p}$ $\left(\mathrm{Jg}^{-1} \mathrm{~K}^{-1}\right)$ are the density and the specific heat of the material, respectively. This differential equation has second order terms, thus requires two boundary conditions to be solved: Dirichlet (imposed temperature $T=T_{b}$ on part of the boundary $\Gamma$ ) and Neumann (imposed flux $q=-k \frac{\partial T}{\partial n}$, with $n$ being the normal vector of the boundary geometry). Other heat transfer phenomena such as convection and radiation are expressed as boundary conditions of the conduction problem. Time $t$ appears as a first order term in Eq. 1 , hence an initial value is enough to solve the system.

The solution of the conduction equation in a threedimensional complex geometry is a challenging problem in many aspects. Computational methods require the discretization of the domain (space and time), which implies the discretization of the equations. Two approaches are commonly used for this purpose: FDM or FEM. In this study, FEM was used as the main technique for treating the spatial domain $\Pi$, because it allows to deal with complex geometries. On the other hand, FDM was applied for the discretization of time (transient scheme).

\section{FEM for spatial discretization of conduction}

FEM provides a solution to partial differential equations through a system of algebraic equations with a finite number of unknowns in the domain (Lewis et al. (2004)). A set of elements with known shape (2D: triangles, quadrilaterals, etc; 3D: tetrahedrons, hexahedrons, etc) is defined, within which the temperature variation is described by a polynomial of first, second or higher order. Hence, the temperature can follow constant, linear, parabolic and other behaviors. The functions that describe this interpolation are the shape functions. For example, given a two-dimensional quadrilateral, the temperature at any point $p$ inside the element can be expressed as:

$$
T(p)=N_{1}(p) T_{1}+N_{2}(p) T_{2}+N_{3}(p) T_{3}+N_{4}(p) T_{4}
$$

where $T_{i}$ is the temperature at node $i$ and $N_{i}$ the correspondent shape function. By replacing the continuous temperature field in Eq. 1 with its discretized form, and minimizing the energy balance using the Galerkin method, a system of equations is defined:

$$
\mathbf{C}\left\{\frac{\partial T}{\partial t}\right\}+\mathbf{K}\{T\}=\{f\}
$$

In this equation, $\mathbf{C}$ is the capacitance matrix (diagonal matrix) and $\mathbf{K}$ is the conductivity matrix (sparse matrix). $\{T\}$ is the vector of unknowns, and $\{f\}$ is the load vector. $\left\{\frac{\partial T}{\partial t}\right\}$ is the vector of temperature derivatives over time, which is not yet discretized. Eq. 4 shows the value of each element of the matrices. Remaining derivatives and integrals are solved using a combination of analytical and numerical methods.

$$
\begin{gathered}
\mathbf{C}_{i j}=\int_{\Pi} \rho c_{p} N_{j} N_{i} d p, \quad \mathbf{K}_{i j}=\int_{\Pi} k \nabla N_{j} \nabla N_{i} d p \\
\{f\}_{i}=-\int_{\Gamma_{q}} N_{i} q d p
\end{gathered}
$$

\section{FDM for time discretization}

The time-temperature derivatives are discretized using a forward difference approach. Using Taylor series, a first order approximation of the derivatives is:

$$
\frac{\partial T_{t_{i}}}{\partial t} \approx \frac{T_{t_{i+1}}-T_{t_{i}}}{\Delta t}
$$

Assuming a linear variation of temperature inside a short timestep length $\Delta t$, it can be said that:

$$
f_{t}=\theta f_{t_{i+1}}+(1-\theta) f_{t_{i}}
$$

Replacing and rearranging Eqs. 5 and 6 in Eq. 3, the following system is obtained:

$$
\begin{gathered}
(\mathbf{C}+\theta \Delta t \mathbf{K})\left\{T_{t_{i+1}}\right\}=(\mathbf{C}-(1-\theta) \Delta t \mathbf{K})\left\{T_{t_{i}}\right\}+ \\
\Delta t\left\{\theta f_{t_{i+1}}+(1-\theta) f_{t_{i}}\right\}
\end{gathered}
$$

The value $\theta=1$ was used in this study, corresponding to an implicit scheme (unconditionally stable):

$$
(\mathbf{C}+\Delta t \mathbf{K})\left\{T_{t_{i+1}}\right\}=\mathbf{C}\left\{T_{t_{i}}\right\}+\Delta t\left\{f_{t_{i+1}}\right\}
$$

$\Delta t$ and $\left\{f_{t_{i+1}}\right\}$ are imposed, while $\left\{T_{t_{i}}\right\}$ is computed in the previous step.

\section{Convection}

A pure FEM treatment of heat transfer does not allow for the calculation of complex air movements within the city. Computational fluid dynamics can be incorporated into the model, but these bring much harder computational challenges and complicate the study. In this work, convection was simplified with the use of the heat transfer coefficient, commonly represented by the letter $h$. This approximation works properly when wind is not dominant in the system, i.e. a calm day is being studied. The equation that represents the heat flux by convection is presented in Eq. 9, which is provided by Newton's law of cooling.

$$
q_{c}=h\left(T-T_{\text {air }}\right)
$$

where $T_{a i r}$ is the air temperature, and $h$ is measured in $W m^{-2} K^{-1}$. 
Fixed air temperature. When $T_{a i r}$ is input data (for example if the air temperature was measured in situ), then convection is treated as a Neumann boundary condition. Two terms are added to the system of Eq. 4:

$$
\mathbf{K}_{i j}^{c o n v}=\int_{\Gamma_{c}} h N_{j} N_{i} d p \quad, \quad\{f\}_{i}^{c o n v}=\int_{\Gamma_{c}} N_{i} h T_{a i r} d p
$$

Free air temperature. When $T_{\text {air }}$ is not known (for example, if the internal air temperature was not measured) it is possible to calculate the interaction between the surface temperature of the boundary and the air. In this way, $T_{\text {air }}$ is a new variable in the system, and an initial air temperature at $t_{0}$ is enough for computing it. Because Cast3m does not support this variation, some coding must be implemented.

A first approach for addressing this problem is the following. A new virtual node is added to the geometry, representing the air mass. All the boundary nodes are connected to the virtual node, and the airsurface interaction is modeled as a conduction problem within the FEM engine. The main advantage of this approach is that, as long as other nonlinearities (such as radiation) are not introduced, the problem remains linear. However, the computational performance for solving the system can be affected because the density factor of $\mathbf{K}$ is increased, and its sparse structure can change (Karimi et al. (2014)).

In this study, a different approach was implemented, based on the techniques used in electric analogy systems (Reinhart and Davila (2016)). In contrast with the method described in last paragraph, the surfaceair interaction can be uncoupled from the FEM engine, and treated as part of the discretization of time with FDM. An iterative solver was used for solving the system of equations at each timestep.

Let us study this interaction for the case of an air mass enclosed in a room with a varying surface temperature. It is important to define the notion of the time constant $\tau$, which is the parameter characterizing the response to a step input of a first-order, linear time-invariant system. The inverse of this constant $\left(s^{-1}\right)$ is:

$$
\frac{1}{\tau}=\frac{h S}{\rho c_{p} V}
$$

where $\rho$ and $c_{p}$ are the density and specific heat of air, $V$ is the volume of the air $\left(\mathrm{m}^{3}\right)$ and $S$ is the surface in contact with the air $\left(\mathrm{m}^{2}\right)$.

Given an initial air temperature and the current internal surface temperatures, the goal is to compute the new air temperature and a new surface temperature. Using Newton's law of cooling, with the assumption that all the internal surfaces have the same $h$ coefficient, the next differential equation is obtained:

$\frac{d T_{\text {air }}(t)}{d t}=-\frac{1}{\tau}\left(T_{\text {air }}(t)-T_{S}(t)\right)$, with a given $T_{a i r}(0)$ where $T_{S}(t)$ is the mean surface temperature at time $t$. Because a FDM is being used, the evolution of internal surface temperature follows a linear behavior within the timestep of length $\Delta t$ :

$$
T_{S}(t)=(1-t / \Delta t) T_{S}^{p r e v}+(t / \Delta t) T_{S}^{c u r}
$$

where $T_{S}^{\text {prev }}$ is the surface temperature of the previous timestep, and $T_{S}^{c u r}$ is the current solution of the iterative solver.

Joining Eqs. 12 and 13, a new differential equation is obtained:

$$
\frac{d T_{a i r}}{d t}=-\frac{1}{\tau} T_{a i r}(t)-\frac{1}{\tau}\left(\frac{T_{S}^{p r e v}-T_{S}^{c u r}}{\Delta t}\right) t+\frac{1}{\tau} T_{S}^{\text {prev }}
$$

This equation has an analytical solution, which simplifies the computational effort. For each timestep, the equation is solved multiple times in the iterative solver, until reaching surface and air temperature convergence. The extension of Eq. 14 for various different $h$ coefficients (for instance for each wall, roof and floor, totaling six surfaces) implies the following re-definition of the time constant: $\frac{1}{\tau}=\sum_{i=1}^{6} \frac{h_{i} S_{i}}{\rho c_{p} V}$.

\section{Adding support for windows}

Electric analogy systems allow for the simulation of windows without increasing the geometrical complexity of the model. In this study, a similar approach was used for adding this features to the FEM system.

Windows have a dual effect on convective heat exchange. In the first place, ventilation and air filtrations imply a direct exchange between external and internal air. In the second, the low thermal inertia of the thin glass present in windows causes a much faster heat flow. Therefore, two components were added to Eq. 12 to account for the effect of windows:

$$
\begin{gathered}
\frac{d T_{a i r}}{d t}=-\frac{1}{\tau}\left(T(t)-T_{S}(t)\right) \\
-\frac{U_{V} A_{V}}{\rho c_{p} V}\left(T_{a i r}(t)-T_{e x t}(t)\right)-\frac{H_{V}}{\rho c_{p} V}\left(T_{a i r}(t)-T_{e x t}(t)\right)
\end{gathered}
$$

In this equation, several terms are introduced. $U_{V}$ is the thermal transmittance of the window $\left(W m^{-2} K^{-1}\right) . A_{V}$ is the surface area of the window. This term is related to the concept of window to wall ratio, which is a measure of the total glazed area with respect to the total envelope area. $H_{V}$ is the heat transmission coefficient due to ventilation $\left(W K^{-1}\right)$ :

$$
H_{V}=\frac{\eta \rho c_{p} V}{3600}
$$

where $\eta$ is the air change rate $\left(A C H, h^{-1}\right)$. Finally, $T_{\text {ext }}$ is the external air temperature, which is a function of time and grows linearly between two timesteps (in a similar way than Eq. 13):

$$
T_{\text {ext }}(t)=(1-t / \Delta t) T_{\text {ext }}^{(i-1)}+(t / \Delta t) T_{e x t}^{(i)}
$$


where $T_{e x t}^{(i-1)}$ and $T_{e x t}^{(i)}$ are the external air temperatures of the previous and current timestep, respectively. For the sake of clarity, a new parameter is defined, which is the inverse of the time constant with respect to the effect of windows in the system:

$$
\frac{1}{\tau_{V}}=\frac{U_{V} A_{V}+H_{V}}{\rho c_{p} V}
$$

Joining Eqs. 15, 16, and 17, a new differential equation is obtained:

$$
\begin{gathered}
\frac{d T_{\text {air }}}{d t}+a T_{\text {air }}+b t-c=0, \text { with a given } T_{\text {air }}(0) \\
\text { where } a=\frac{1}{\tau}+\frac{1}{\tau_{V}} \\
b=\frac{1}{\tau}\left(\frac{T_{S}^{\text {prev }}-T_{S}^{c u r}}{\Delta t}\right)+\frac{1}{\tau_{V}}\left(\frac{T_{e x t}^{(i-1)}-T_{\text {ext }}^{(i)}}{\Delta t}\right) \\
c=\frac{1}{\tau} T_{S}^{\text {prev }}+\frac{1}{\tau_{V}} T_{\text {ext }}^{(i-1)}
\end{gathered}
$$

The analytical solution of this equation is:

$$
T_{a i r}(t)=\frac{c-b t}{a}+\frac{b-e^{-a t}\left(-T_{a i r}(0) a^{2}+c a+b\right)}{a^{2}}
$$

\section{Long wave radiation}

Thermal studies of cities must address long wave radiative exchange accurately because temperature differences between the involved elements can be large. In particular, low sky temperatures play a major role in the urban cooling process (Morakinyo et al. (2017)).

Assuming that all the surfaces are black bodies (their emissivity value is 1), the radiative flux at a given point on the boundary is expressed by:

$$
q_{l w}=\sigma\left(T^{4}-T_{r}^{4}\right)
$$

where $T_{r}$ is the mean temperature of the environment visible from the point, and $\sigma$ is the Stefan-Boltzmann constant $\left(\approx 5.670373 \times 10^{-8} \mathrm{Wm}^{-2} \mathrm{~K}^{-4}\right)$.

With the presence of radiation, the FEM system becomes non-linear. The computational simulation of radiative boundary conditions works in a similar way than a convective flux, but adding an iterative scheme to solve the non-linearity:

$q_{l w}=h_{r a d}\left(T-T_{r}\right)$, with $h_{r a d}=\sigma\left(\tilde{T}^{2}+T_{r}^{2}\right)\left(\tilde{T}+T_{r}\right)$

where $T$ is the temperature to be found, and $\tilde{T}$ is result of the previous iteration. When the iterative process converges, $T=\tilde{T}$, and thus Eqs. 20 and 21 are the same. The most complicated stage of each iteration is finding the mean visible environment temperature $T_{r}$, which involves the use of form factors:

$$
T_{r}=\left(E_{i} / \sigma\right)^{\frac{1}{4}}, \text { with } E_{i}=\sum_{j=1}^{N} F_{i j}\left(\sigma \tilde{T}_{j}^{4}\right)
$$

Because the city geometry is static throughout the entire simulation, matrix $\mathbf{F}$ can be pre-computed and stored. Following Aguerre et al. (2017), F can be stored in sparse format to avoid excessive memory usage and to accelerate the sum in Eq. 21. This enables refined geometries (composed of tens of thousands of elements) to be processed in regular desktop PCs. In this study, the density factor of the involved matrix $\mathbf{F}$ were studied, and the results are presented in the experimental section.

\section{Short wave radiation}

The low density factor of $\mathbf{F}$ was also exploited to compute short wave radiation, which is the radiation emitted by the sun and sky that reaches the surfaces after a limited number of scene bounces. The assumption of working with diffuse concrete materials allows use of the radiosity method for computing irradiance flux over the surfaces of the city. The following linear system must be solved:

$$
(\mathbf{I}-\mathbf{R F}) J=\xi
$$

where $\mathbf{I}$ is the identity matrix, $\mathbf{R}$ a diagonal matrix of short wave reflectivity coefficients, $J$ the radiosity values and $\xi$ the emission. The strategy described at Aguerre et al. (2017) was used to compute climatebased radiation with multiple reflections. After the radiosity values $J$ are computed for each element, the absorbed radiation is inputted as an imposed flux (Neumann boundary condition) in the right hand side of Eq. 3.

\section{Test case and model}

The modifications proposed in this study were evaluated using the test case described in Aguerre et al. (2018), which is summarized in this section.

A measurement campaign was carried out in Bayonne, France $\left(43.48 \mathrm{~N}^{\circ}\right)$, in Rue des Tonneliers. A set of thermograms were taken on April $23^{\text {rd }} 2017$ using an infrared FLIR B200 camera. The idea was to maximize the amount of physical information contained in the pictures, thus the street was captured in perspective (Figure 1). The test case used a simplified geometry composed of four boxes that represent the set of buildings captured in the thermograms. The thickness of walls and roofs was $0.18 \mathrm{~m}$. Material properties used are presented in Figure 2.

Regarding environmental conditions, several parameters were experimentally measured, whereas others were modeled or estimated. A clear sky day was selected for the imaging campaign. Very low wind values were measured in situ, allowing for the estimation of a proper value for the external $h$ coefficient, which was set to $10 \mathrm{Wm}^{-2} \mathrm{~K}^{-1}$. The interior $h$ coefficients were extracted from the French thermal regulation (CSTB (2012)): 0.7 for the ground, 2.5 for vertical walls, and 5 for the roof (values in $W m^{-2} K^{-1}$ ). Outside air temperature (see Figure 5) and relative humidity were measured in situ. 


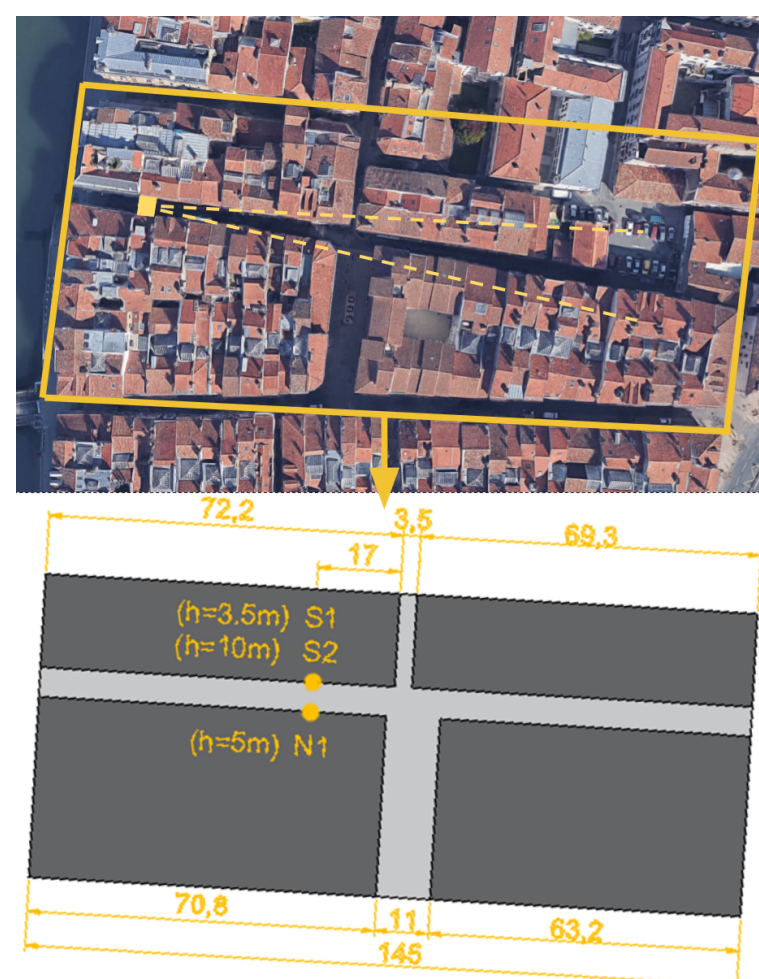

Figure 1: Camera location and model description of the test case. The square and dashed lines indicate the position of the thermal camera and the perspective angle. S1, S2 and N1 are points selected for the study.

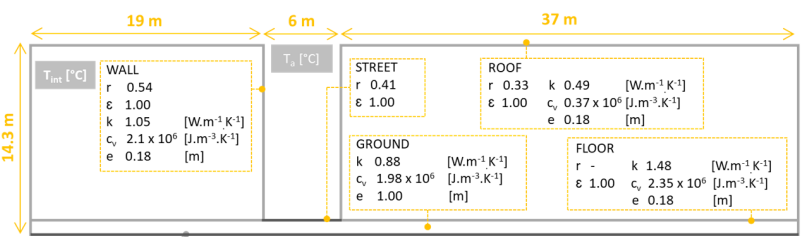

Twist

Figure 2: Material properties of the test case. Extracted from Aguerre et al. (2018).

Global horizontal irradiance was measured in a weather station located $3.4 \mathrm{~km}$ away from the experiments location. Using this data, direct and diffuse irradiances were modeled using Liu-Jordan clear sky model Liu and Jordan (1960). Perez All-weather sky model (Perez et al. (1993)) was used to compute short wave irradiances, using a sky dome geometry divided into 2400 elements (Beckers and Beckers (2014)).

Infrared horizontal irradiance and sky temperatures were modeled using Angström formula (Ångström (1915)). Sky emissivity and directional temperatures were modeled based on the work described by Nahon (2017). A sky dome geometry discretized into 130 elements was used for computing long wave radiation.

Data was extracted from Meteonorm (Remund et al. (2010)) global meteorological database to estimate the ground temperature at $1 \mathrm{~m}$ deep $\left(11.1^{\circ} \mathrm{C}\right)$, value that can be considered constant for a day period.

The internal air temperature of buildings was proposed to be fixed at $20^{\circ} \mathrm{C}$, which was highlighted as a questionable supposition by the authors. This as- sumption was changed in the study presented in this paper, where these temperatures were also simulated using the method described above. Results can be observed in the next section.

The implementation of this test case in a FEM engine like Cast3m requires meshing the geometry to obtain a discretized version of the problem. The geometry was meshed using hexahedrons of linear shape functions, with four layers of elements along the thickness of each wall. The boundary elements are quadrilaterals of area $1.5 \mathrm{~m} \times 1.5 \mathrm{~m}$ (see Figure 3 ). The total model is composed of four boxes, street, ground, and sky dome for long wave radiation. The number of volume elements is 113580, and the number of boundary elements is 28395 .

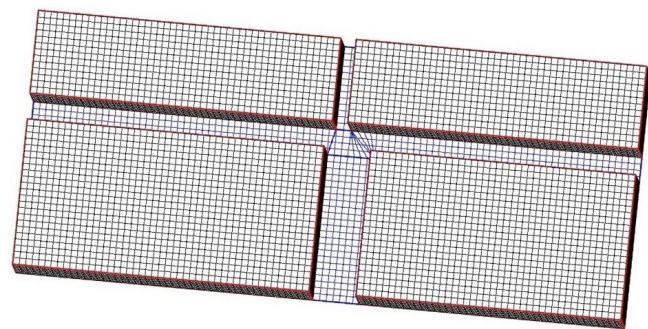

Figure 3: Mesh representing the environment of Rue des Tonneliers, used for the FEM simulation.

Because the series of thermograms were taken during one day, the aim of this study was to simulate 24 hours. Accurate simulation results depend highly on initialization conditions. A strategy commonly used in electric analogy systems was adopted: the simulated period was repeated several times. The results for the first day differ considerably from the second, but they converged for the third day. Hence, a three day repetition was enough to obtain the initial temperatures of the surfaces. The results presented in the next section correspond to the third day of simulation.

\section{Results and discussion}

Table 1 shows the thermography campaign and the correspondent simulated results. Temperature patterns are similar in both experimental and simulated images. Higher accuracy is reached for the wall that receives direct sunlight through most of the day. In the other wall, as well as in the street, temperatures are overestimated due to lower short wave radiation values, which can be explained by the geometrical simplifications (flat roofs, no overhangs, etc) . It is important to highlight that the red parts observed at 16:00 in the thermograms correspond to wooden carpentry and joinery, elements that are not present in the simulation.

The simulation with free internal air temperature is more accurate than the one with fixed air at $20^{\circ} \mathrm{C}$. Because the difference between both set of images is not easy to appreciate, an image showing the absolute difference (from $0^{\circ} \mathrm{C}$ to $1.5^{\circ} \mathrm{C}$ ) is presented. The 

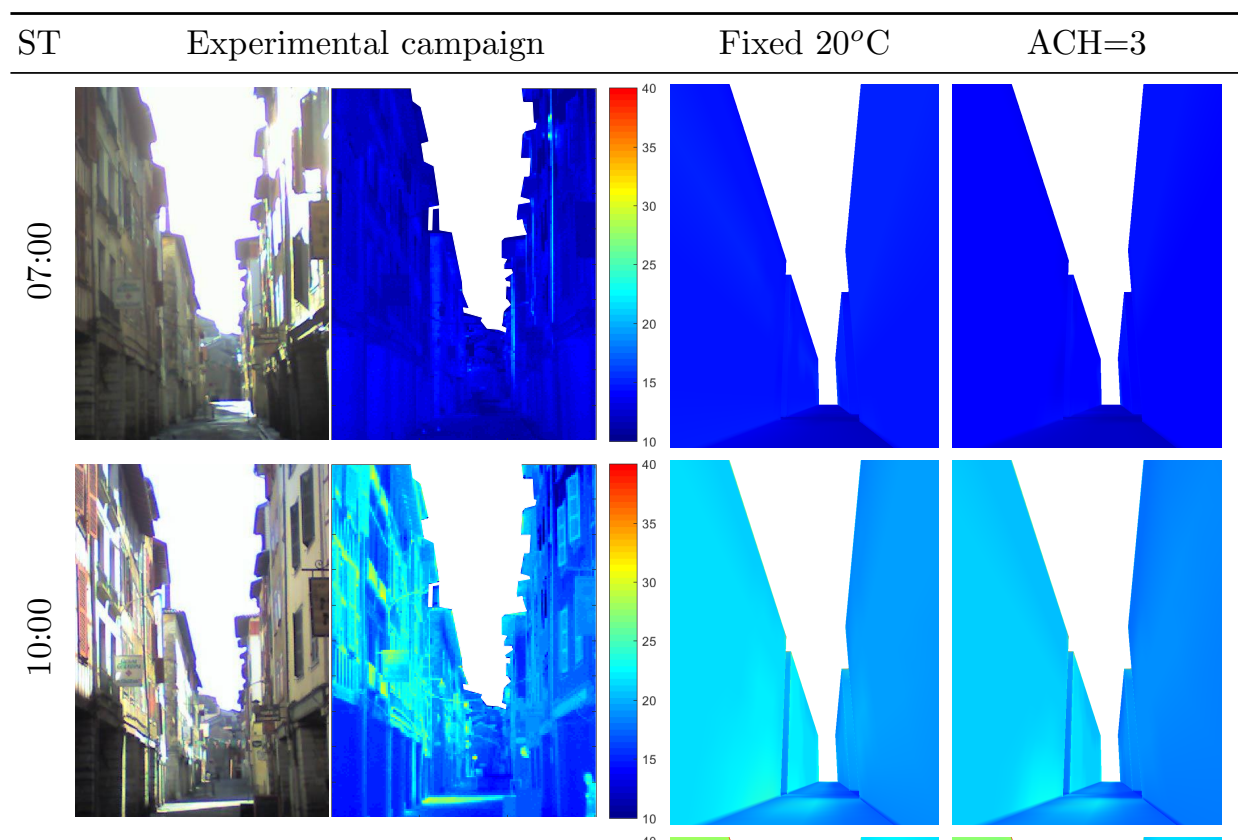

Difference
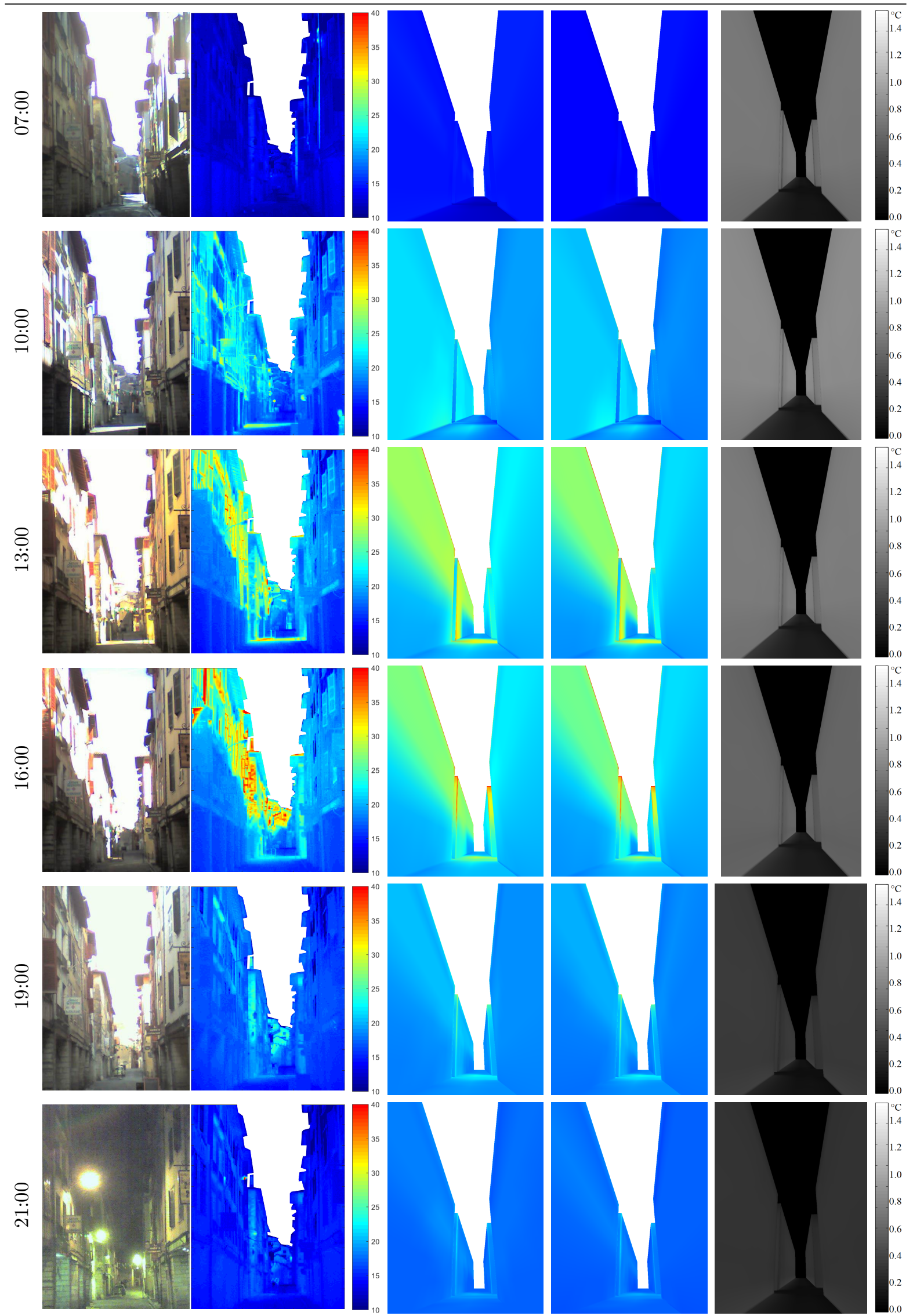

Table 1: Time-lapse (in solar time ST) of the photographs, thermograms, and corresponding simulated results for fixed and free internal air temperature. Column 5 corresponds to the absolute difference between the two simulated thermograms in columns 3 and 4. 


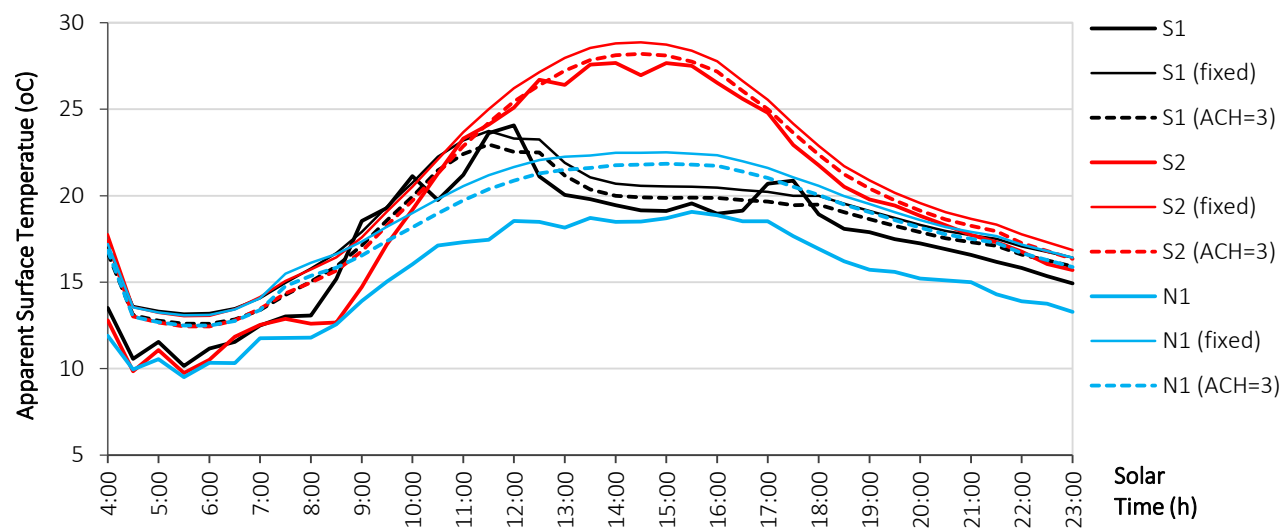

Figure 4: Comparison between simulated and measured temperatures at the selected points.

impact of the internal air temperature on the surface temperature of the walls is greater than on the street, due to conduction along the thickness of walls. To enable a better evaluation of the results, three points were selected in the geometry (see Figure 2). The evolution of the experimental apparent surface temperature was obtained by selecting pixels on the thermograms and matching their color with the respective temperature. These results are presented in Figure 4, along with the correspondent simulations. The results with $\mathrm{ACH}=3$ are closer to the experimental data than those with fixed air temperature.

The modifications of FEM implemented in this study allow for the estimation of internal air temperature. Figure 5 shows the evolution of the internal air temperature using different configurations of $\mathrm{ACH}$, along with the external air temperature. The three plots correspond to the internal results of one of the studied blocks. The results showed no significant differences for the rest of the blocks. Thermal inertia of air is observed, where the minimum of the three curves is always hotter than the minimum external temperature. An analogous behavior is shown for the maximum temperature. Larger $\mathrm{ACH}$ values implied a greater similarity between the evolution of internal and external temperatures.

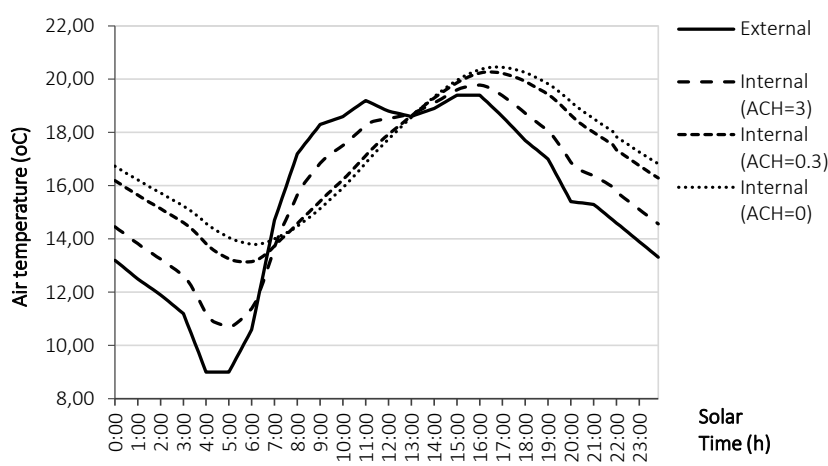

Figure 5: Evolution of external and internal air temperatures using different air change rates.

The results shown in Figure 5 explain the differences observed in column 5 of Table 1 . An internal air temperature fixed at $20^{\circ} \mathrm{C}$ was far away from the com- puted one, where the maximum gap with $\mathrm{ACH}=3$ is observed around 05:00 $\left(\approx 9^{\circ} C\right)$. Due to the thermal inertia of the wall, this difference takes some time to be reflected on the outside surface temperature, which reached its maximum difference around 10:00.

\section{Computational performance}

The simulations presented in this study were executed on a standard desktop PC (core i7 processor and 16GB of RAM memory). The code implemented for the computation of internal air temperatures did not affect the computational performance of the model, because an iterative solver was already being used to account for the non-linearities associated with radiation. The number of iterations needed to converge in each timestep is the same with fixed and varying internal air temperatures.

The memory consumption of Cast3m depends highly on the size of the geometry (number of elements). In particular, matrix $\mathbf{F}$ from Eq. 23 is a square matrix of dimensions $\approx 23 k \times 23 k$. Using full matrix representations, which is the current Cast3m implementation, the memory requirement to store $\mathbf{F}$ was $\approx 4 G B$. Because of the geometrical properties of the scene, the density factor of $\mathbf{F}$ is equal to $4.16 \%$, which justified the use of sparse representations. The memory consumption was reduced to $331 M B$, and the radiation step was considerably accelerated. Table 2 shows results for the entire FEM model.

\begin{tabular}{|c|c|c|}
\hline Version & $\begin{array}{c}\text { Execution time } \\
\text { (minutes) }\end{array}$ & $\begin{array}{c}\text { Max. memory } \\
\text { consumption (GB) }\end{array}$ \\
\hline Original Cast3m & 572 & 4.6 \\
\hline Sparse matrix & 61 & 0.9 \\
\hline
\end{tabular}

Table 2: Execution time and memory usage details.

Around $9 \times$ speedup was reached in total execution times. The memory consumption was reduced by a factor of 5.1.

Sparse form factors matrices enable dealing with larger geometries, which can be an important results for future simulations. For example, the size of the geometry can easily scale to hundreds of thousands elements if a higher level of detail is modeled, and/or if a larger zone wants to simulated. 


\section{Conclusion}

In this study, a set of thermograms were simulated using FEM, and the results were compared to an experimental thermography campaign. The computational techniques used were presented, and two improvements regarding convective and radiative boundary conditions were implemented in Cast3m.

The results showed that a simplified geometry can generate accurate results if the computational model is highly precise. In particular, using free internal air temperatures increased the realism of the simulation, reaching accurate results when compared to experimental data. In periods where occupants do not make use of artificial temperature conditioners, measuring the air temperature can also serve as trustful information about thermal comfort.

Furthermore, the computational performance of the algorithm was optimized by using sparse matrix representations for storing form factors. The entire simulation was executed in a standard desktop PC in reasonable times, enabling the simulation of much longer periods of time.

\section{Acknowledgments}

This work was partially funded by project FSE_1_2017_1_144731 from Agencia Nacional de Investigación e Innovación (ANII, Uruguay).

\section{References}

Aguerre, J. P., E. Fernandez, G. Besuievsky, and B. Beckers (2017). Computing urban radiation: A sparse matrix approach. Graphical Models 91, 111.

Aguerre, J. P., R. Nahon, E. Garcia-Nevado, C. La Borderie, E. Fernández, and B. Beckers (2018). A street in perspective: Thermography simulated by the finite element method. Building and Environment.

Ångström, A. K. (1915). A study of the radiation of the atmosphere: based upon observations of the nocturnal radiation during expeditions to Algeria and to California, Volume 65. Smithsonian Institution.

Asawa, T., A. Hoyano, and K. Nakaohkubo (2008). Thermal design tool for outdoor spaces based on heat balance simulation using a 3d-cad system. Building and Environment 43(12), 2112-2123.

Beckers, B. and P. Beckers (2014). Sky vault partition for computing daylight availability and shortwave energy budget on an urban scale. Lighting Research E3 Technology 46(6), 716-728.

Beckers, B. and E. Garcia-Nevado (2018). Urban planning enriched by its representations, from perspective to thermography. Springer International Publishing.

Charras, T. and F. Di Paola (2011). Utiliser cast3m: la procédure pasapas. Documentation Cast3M.

CSTB Editions (2012). Régles Th-U - Fasicule 4: Parois opaques.

Fraisse, G., C. Viardot, O. Lafabrie, and G. Achard (2002). Development of a simplified and accurate building model based on electrical analogy. Energy and buildings 34(10), 1017-1031.

Huttner, S. and M. Bruse (2009). Numerical modeling of the urban climate-a preview on envi-met 4.0. In 7th international conference on urban climate ICUC-7, Yokohama, Japan, Volume 29.

Idczak, M., D. Groleau, P. Mestayer, J.-M. Rosant, and J.-F. Sini (2010). An application of the thermoradiative model solene for the evaluation of street canyon energy balance. Building and Environment 45(5), 1262-1275.

Karimi, R., A. A. Akhtari, and O. Seyedashraf (2014). Implementation of the skyline algorithm in finiteelement computations of saint-venant equations. Journal of Applied Research in Water and Wastewater 1(2), 61-65.

Kramer, R., J. Van Schijndel, and H. Schellen (2012). Simplified thermal and hygric building models: A literature review. Frontiers of architectural research 1(4), 318-325.

Lewis, R. W., P. Nithiarasu, and K. N. Seetharamu (2004). Fundamentals of the finite element method for heat and fluid flow. John Wiley \& Sons.

Liu, B. Y. and R. C. Jordan (1960). The interrelationship and characteristic distribution of direct, diffuse and total solar radiation. Solar energy 4 (3), $1-19$.

Lü, X. (2002). Modelling of heat and moisture transfer in buildings: I. model program. Energy and buildings 34 (10), 1033-1043.

Morakinyo, T. E., K. K. C. Dahanayake, E. Ng, and C. L. Chow (2017). Temperature and cooling demand reduction by green-roof types in different climates and urban densities: A co-simulation parametric study. Energy and Buildings 145, 226-237.

Nahon, R. (2017). Modélisation des échanges radiatifs à l'échelle urbaine pour un urbanisme bioclimatique. Ph. D. thesis, Lille 1.

Nielsen, T. R. (2005). Simple tool to evaluate energy demand and indoor environment in the early stages of building design. Solar Energy 78(1), 73-83.

Perez, R., R. Seals, and J. Michalsky (1993). All-weather model for sky luminance distribution - preliminary configuration and validation. Solar energy 50 (3), 235-245.

Reinhart, C. F. and C. C. Davila (2016). Urban building energy modeling-a review of a nascent field. Building and Environment 97, 196-202.

Switzerland, Tech. Rep (2010). Meteonorm handbook part I: software. 